\title{
Research on the Remote Deployment Design of OTN Electrical Racks
}

\author{
Tianpu Yang ${ }^{(凶)}$, Junshi Gao, Haitao Wang, Guangchong Dai, and Rui Zhai \\ China Mobile Group Design Institute Co., Ltd., Beijing 10080, China \\ yangtianpu@cmdi.chinamobile.com
}

\begin{abstract}
The rapid development of $4 \mathrm{G}$ and multimedia services drives the exponential increase of the demand for transmission bandwidth. The OTN technology therefore emerges. In recent years, the number of OTN devices in backbone and core equipment rooms has increased sharply. However, due to factors such as equipment room planning, air conditioner, and power supply, new electrical racks cannot be installed in the same equipment room as original optical racks during OTN expansion of 80-wavelength systems. The remote deployment of OTN electrical racks has certain impact on OTN system indicators, OM/OD, and OTU optical-layer parameters. This document analyzes the factors that are affected by the remote deployment of OTN electrical racks, creates simulation models based on scenarios, and provides suggestions on the remote deployment design of OTN electrical racks.
\end{abstract}

Keywords: OTN $\cdot$ Capacity expansion $\cdot$ Remote deployment

\section{Background}

\subsection{Current Situation}

The rapid development of $4 \mathrm{G}$ and multimedia services drives the exponential increase of the demand for transmission bandwidth. The OTN technology therefore emerges. Especially in recent years, the number of OTN devices in China Mobile's backbone and core equipment rooms has increased sharply. For example, the inter-province backbone transport network has been constructed from phase 8.2 to phase 12 , covering over 2,000 electrical racks on the entire network. However, due to factors such as equipment room planning, air conditioners, and power supplies, new 100G $\times 80$-wavelength OTN systems cannot be installed in the same equipment room as the original optical racks during capacity expansion. As a result, the OTN electrical racks need to be remotely deployed.

\subsection{Inevitability of Remote Electrical Rack Deployment}

As networks develop rapidly, OTN devices are no longer integrated but separated. As the number of OTN devices increases sharply, the power consumption in equipment rooms increases rapidly, and the equipment room footprint is prominently insufficient. It is inevitable that OTN electrical racks are remotely deployed during capacity expansion. 
Optical and Electrical Racks Have Been Separated. As shown in Fig. 1, the industry's current AWG demultiplexing/multiplexing mode at the optical layer consists of optical-layer boards such as AWG multiplexers/demultiplexers, comb filters, and optical amplifiers (OAs). These boards are connected using optical fibers rather than backplane buses. The backplanes of the optical-layer boards only need to be responsible for power supply and communication control. Therefore, the required structure is simple.

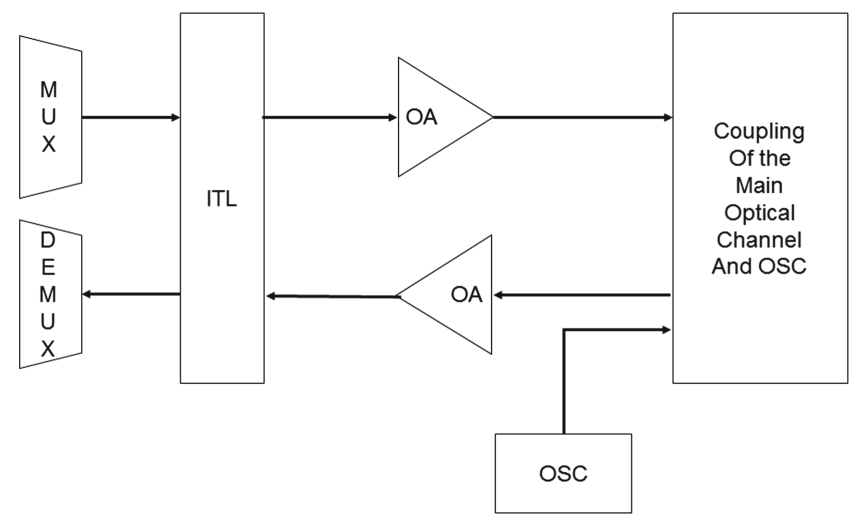

Fig. 1. Main optical-layer architecture of AWG multiplexers/demultiplexers

The OTN architecture provides an over $64 \mathrm{Tbit} / \mathrm{s}$ switching capability, over $1 \mathrm{Tbit} / \mathrm{s}$ in each slot. The backplanes are powerful in grooming. If optical-electrical integration is still used, slot waste will cause loss of the electrical-layer grooming capability. To address this issue, optical-electrical separation can be used so that optical and electrical racks can play their respective advantages to achieve the optimal combination of performance and costs.

\section{Rapid Development of Transmission Requirements Brings Sharp Increase of OTN Quantity. The rapid development of $4 \mathrm{G}$ and broadband has driven the rapid increase of the traffic of transmission networks, which will be further boosted by $5 \mathrm{G}$ and $4 \mathrm{~K}$ appli- cations. Currently, a provincial backbone network has three to four planes. Optical racks deployed in one cabinet at core sites support two to four optical directions. Considering that $50 \%$ wavelengths need to be added and dropped, four to eight OTN electrical racks need to be installed. The more services, the more optical directions and systems, and the more OTNs.}

\section{Power Consumption Increase of the Entire OTN Device Requires More Cabinets} for Installing OTN Devices. Service development drives technology improvement. The improvement of cross-connect capacity and integration leads to the continuous increase of the power consumption of the entire device. After the single-wavelength bandwidth of OTN reaches $100 \mathrm{G}$, the single-bit power consumption is continuously reduced, but the device capacity increases from less than $5 \mathrm{Tbit} / \mathrm{s}$ to over $20 \mathrm{Tbit} / \mathrm{s}$, causing the rapid power consumption increase of the entire device. However, the heat 
dissipation and maintenance of the current operators' equipment rooms are outdated. The heat dissipation conditions of transmission equipment rooms cannot support higher power consumption. Therefore, boards need to be split into multiple electrical cabinets. As a result, a large number of OTN devices are installed in more OTN electrical racks. For example, a fully configured Huawei OSN 9800 U64 subrack cannot be installed in an equipment room supporting a maximum power consumption of $5000 \mathrm{~W}$. Four U32 subracks need to be installed instead.

Poor Equipment Room Planning, Making Capacity Expansion Restricted by Cabinet Space and Power Supplies. Some equipment rooms are not well planned. For example, one equipment room houses multiple types of devices, such as transmission devices and IP devices, or houses devices of the national backbone network, provincial backbone network, and local metro network. In addition, the development among some sites is unbalanced. For example, a provincial core site accesses multi-layer services at the same time, such as those from the national backbone, provincial backbone, and local metro networks. There are five to six rings and multiple optical directions, requiring more than 50 OTN electrical cabinets. Therefore, as services develop, cabinet space or power supply becomes insufficient. As a result, new equipment rooms must be constructed. After capacity expansion, the electrical racks can only be remotely deployed.

\section{Implementation Mode and Impact}

The remote deployment of OTN devices prolongs the distance between OTU and OM/OD boards, increasing the attenuation and affecting the receive optical power of OTU boards. For example, if a pair of multiplexer/demultiplexer is added for optical-layer regeneration between the existing optical layer and a remote OTN electrical rack, system performance indicators will be affected. In addition, the remote electrical racks cause inconvenience to fiber patch cord maintenance.

\subsection{Implementation Modes}

Based on factors such as environments and distances, optical and electrical racks can be remotely deployed in the ways described in the following sections.

Direct Connection Using Optical Fibers/Cables. Direct connection using optical fibers or cables is the most common mode, which is used in the following scenarios:

- An electrical rack and an optical rack in the same equipment room are directly connected using an optical fiber routed along the fiber trough. In this case, fiber attenuation can be ignored.

- An electrical rack and an optical rack in different equipment rooms on the same floor are directly connected through an optical fiber. In this case, fiber attenuation is large.

- An electrical rack and an optical rack on different floors or in different buildings are connected using ODFs. In this case, both fiber attenuation and connector attenuation must be considered (Fig. 2). 


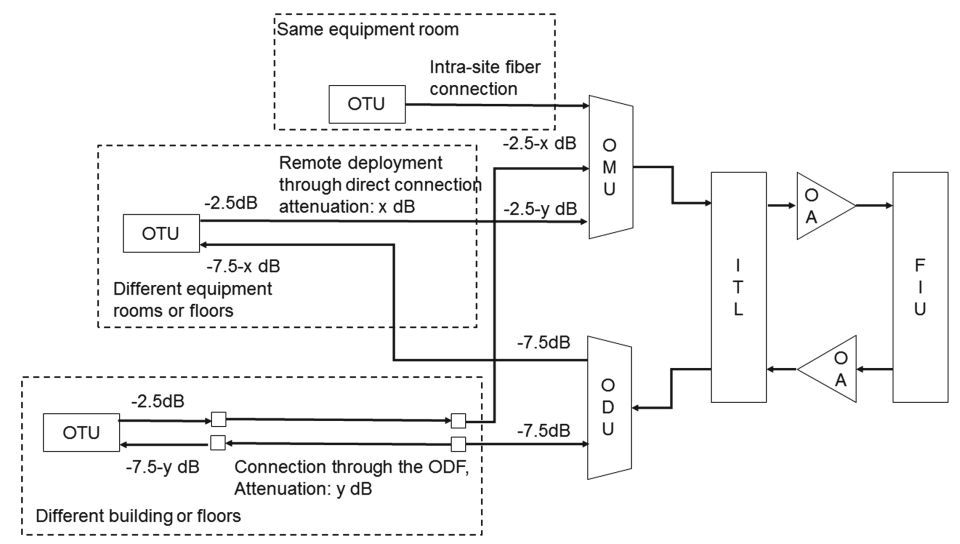

Fig. 2. Direct connection between OTU boards and multiplexers/demultiplexers using optical fibers

Devices will generate alarms and cannot function properly in the following direct connection scenarios:

- The remote electrical rack and the original optical rack are deployed in different buildings or at different sites. The fiber attenuation is greater than $2.5 \mathrm{~dB}$.

- The optical fibers between the remote rack and the original rack cannot be routed properly, or the number of optical fibers is insufficient.

Adding OMSs. Optical multiplex sections (OMSs) are added to connect the original optical rack and the remote electrical rack. To be specific, a pair of optical racks that share a pair of fiber cores is added, and the new optical racks and original racks are connected using LC-LC fiber patch cords, as shown in Fig. 3.

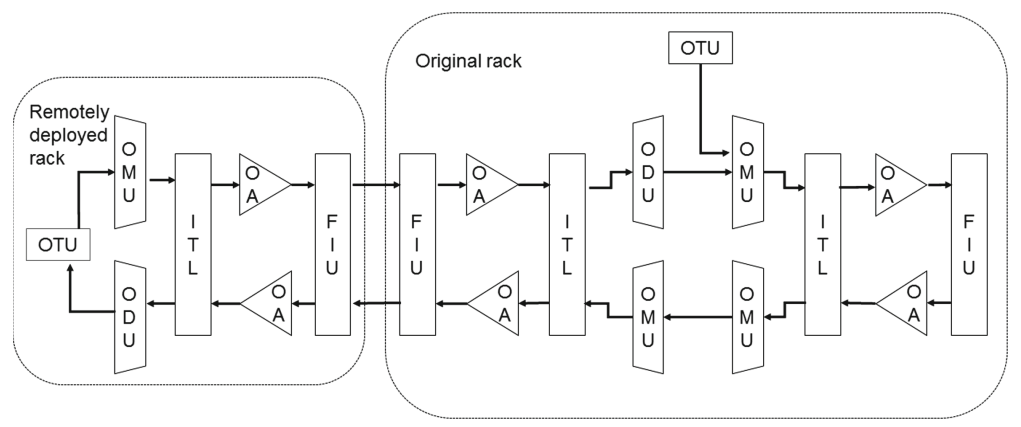

Fig. 3. Adding OMSs

Adding WSS Boards for Demultiplexing/Multiplexing. A pair of WSS board is added between the original optical rack and the remote electrical rack for demultiplexing/multiplexing, as shown in Fig. 4. 


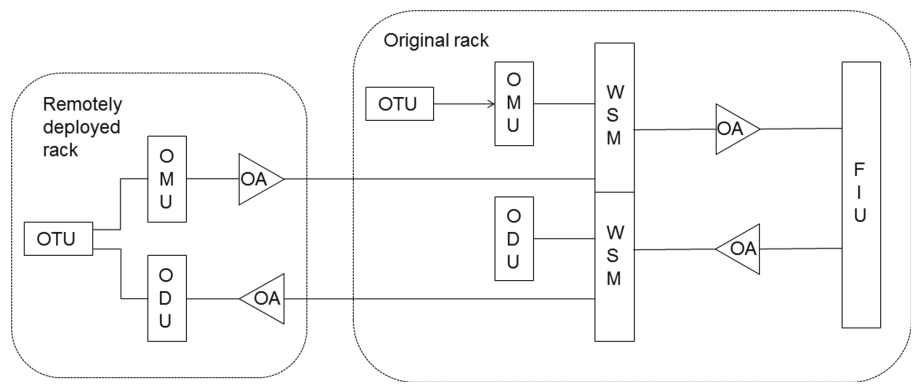

Fig. 4. Adding WSS boards for demultiplexing/multiplexing

\subsection{Impact on System OSNR}

The remote deployment of electrical racks has certain impact on system OSNR:

- In direct connection mode, the attenuation caused by the remote deployment of electrical racks occurs before the OTU board. As a result, the received signals and noise of the OTU board are amplified or reduced at the same level, without affecting the OSNR.

- Adding an OMS to separate optical and electrical racks is equivalent to adding an OMS with $10 \mathrm{~dB}$ loss between them, which decreases the OSNR by 0.3 to $1 \mathrm{~dB}$.

- Adding WSS boards to separate optical and electrical racks introduces fixed noise, which has impact on the OSNR.

\subsection{Impact on the Receive Optical Power of the OM/OD or OTU Boards}

The optical and electrical racks of OTN devices are separated, and the electrical racks are remotely deployed. This affects the receive optical power of the OM/OD and OTU boards.

Direct Connection Using Optical Fibers/Cables. In the direct fiber connection mode, the input optical power of the OM port and the receive optical power of the OTU board decrease. The attenuation less than $2.5 \mathrm{~dB}$ has no impact on the system. When the attenuation is greater than $2.5 \mathrm{~dB}$, the input optical power of the OTU board becomes excessively low. As a result, an alarm may be reported.

- Permitted attenuation between the wavelength-dropping port of the demultiplexer and the input port of the OTU board: The output single-wavelength optical power of the $\mathrm{OA}$ is $1 \mathrm{dBm}$, and the insertion loss of the demultiplexer is less than $6.5 \mathrm{~dB}$. Considering the flatness, the output optical power of the wavelength-dropping port of the demultiplexer is $-7.5 \mathrm{dBm}$. It is recommended that the receive optical power of the optical port on the OTU board be greater than or equal to $-10 \mathrm{dBm}$. Otherwise, an alarm indicating abnormal optical power may be generated. Therefore, the permitted attenuation between the demultiplexer and the OTU board must be less than $2.5 \mathrm{~dB}$. 
- Permitted attenuation between the output port of the OTU board and the multiplexer: The transmit optical power of the OTU board is $-2.5 \mathrm{dBm}$, the insertion loss of the multiplexer is less than $8 \mathrm{~dB}$, and the gain of the $\mathrm{OA}$ on the transmit side is about $20 \mathrm{~dB}$. The single-wavelength output optical power of the $\mathrm{OA}$ is $1 \mathrm{dBm}$. Assume that the minimum single-wavelength input optical power is $-19 \mathrm{dBm}$. Considering the impact of $3 \mathrm{~dB}$ flatness, the permitted attenuation is calculated as follows: $-2.5-8-3$ $-(-19)=5.5 \mathrm{~dB}$.

Adding OMSs. The input optical power at the $\mathrm{OM}$ is decreased to $-7.5 \mathrm{~dB}$. The impact on the system optical power is like that optical signals pass through one more OTM site. Since OAs are added, there is no impact on the receive optical power of OTU boards.

Adding WSS Boards for Demultiplexing/Multiplexing. In this mode, OAs are added. The gain of the OAs and the VOA at the input ports of the OAs are adjusted to achieve the optimal optical power. The impact on the optical power at the receive end of the OM/OD and OTU boards does not need to be considered. Only the impact on the OSNR needs to be considered.

\subsection{Other Impacts}

The remote deployment of electrical racks also has the following impacts:

- For fiber routing across floors or buildings, each OTU port requires two fibers. For the remote deployment of electrical racks in an 80-wavelength system, 160 fibers are required.

- Fault locating: Once a remote optical fiber is faulty, it is difficult to locate and rectify the fault. In this case, the faulty optical fiber needs to be re-routed and replaced.

- Attenuation caused by fiber aging: When the remote deployment distance is long, the impact of fiber attenuation caused by aging and increased connector attenuation must be considered.

\section{Applicability Analysis}

\subsection{Advantages and Disadvantages}

Direct Connection Using Optical Cables. The mode of connecting remotely deployed OTN electrical racks using optical cables has the following advantages:

- Optical cables have a high protection level and strong tension and compression resistance capabilities.

- Optical cables have low requirements on terrain. They can be buried underground or routed through pipes.

- After the deployment is complete, the attenuation of the optical cables changes slightly and is not affected by future construction.

- Optical cables can be repaired once they are broken. 
- However, the mode of connecting remotely deployed OTN electrical racks using optical cables has the following disadvantages:

- Optical cables have high costs and the construction period is long.

- ODFs are required to connect racks, which increases the connector attenuation.

In summary, this mode requires that optical cables have high reliability and deployment flexibility. The attenuation caused by the optical cables and ODFs is within the permitted range, which has no impact on the OSNR. Fiber cores need to be reserved to prevent abnormal attenuation of some fiber cores.

Direct Connection Using Optical Fibers. The mode of connecting remotely deployed OTN electrical racks using optical fibers has the following advantages:

- Optical fibers can be used to directly connect racks without ODFs, and extra connector loss does not need to be considered.

- The cost is lower than that of optical cables.

However, the mode of connecting remotely deployed OTN electrical racks using optical fibers has the following disadvantages:

- Optical fibers have a poor protection capability and require dedicated cable troughs.

- Optical fibers may need to be customized based on required lengths.

- If an optical fiber is faulty, a new optical fiber is required to replace the faulty one.

In this mode, bundle optical fibers or armored optical fibers with an enhanced protection capability are required for direct connections, avoiding connector insertion loss. If the attenuation is within the permitted range, the OSNR will not be affected. If the attenuation is beyond the permitted range, the direct fiber connection mode cannot be used.

Adding OMSs. After the OMSs are added on the transmission network, the system OSNR will decrease, but the intra-site optical power does not need to be considered.

\subsection{Scenario Analysis}

Solutions vary depending on the scenarios where the OTN electrical racks are remotely installed.

Scenario 1: The OSNR margin of the system is large, and the equipment rooms housing the optical and electrical racks are far from each other.

In this case, the attenuation may easily exceed the threshold. Since the OSNR margin of the system is large, adding OMSs is the optimal mode. In the engineering design, the impact on the OSNR must be considered. The intra-site fiber attenuation and construction impact do not need to be considered.

Scenario 2: The OSNR margin of the system is small, and the equipment rooms housing the optical and electrical racks are far from each other. 
In this case, the attenuation of the fibers for direct connections usually exceeds the threshold. So the direct fiber connection mode cannot be used. If OMSs are added, the OSNR margin of the system will be affected and the system performance will deteriorate. Therefore, this mode is not recommended. In this scenario, the space and layout of the equipment rooms need to be adjusted, or the planning and design need to be modified so that the optical and electrical racks are placed in the same equipment room.

Scenario 3: The OSNR margin of the system is large, and the equipment rooms housing the optical and electrical racks are near to each other.

The attenuation in this scenario can be controlled within the required range. The OSNR margin of the system is large. After OMSs are added, the system has sufficient margin. Both modes are applicable. An optimal mode can be selected based on other relevant factors. If the optical and electrical racks are deployed on different floors or two adjacent buildings and fiber troughs are available, the direct fiber connection mode is preferred. Optical and electrical racks are deployed in different buildings and areas (for example, across a street), there is no fiber trough, but there is space for optical racks in the equipment room. In this scenario, it is recommended that OMSs be added for remote deployment.

Scenario 4: The OSNR margin of the system is small, and the equipment rooms housing the optical and electrical racks are near to each other.

The OSNR margin is small, and OMSs cannot be added for remote deployment. In this scenario, the direct fiber connection mode is recommended so that the attenuation is controlled within the required range.

\section{Test Models and Simulation}

\subsection{Scenario-Based Modeling}

Based on the preceding analysis, this section builds simulation models for scenarios with different OSNR margins in the OTN system. The simulation results are obtained based on the impact of different remote deployment modes on the OSNR.

Model with a large OSNR margin: There are eight OA spans between OTM sites. Each span is $80 \mathrm{~km}$ long, and the attenuation is $24 \mathrm{~dB}$.

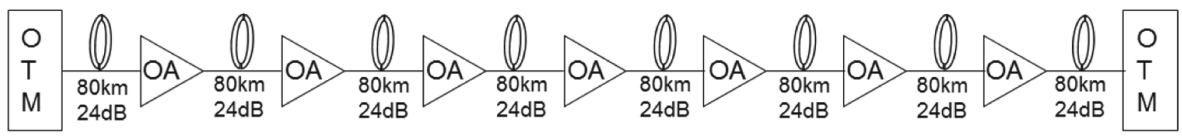

Model with a small OSNR margin: There are eight OA spans between OTM sites. Each span is $80 \mathrm{~km}$ long, and the attenuation is $28 \mathrm{~dB}$.

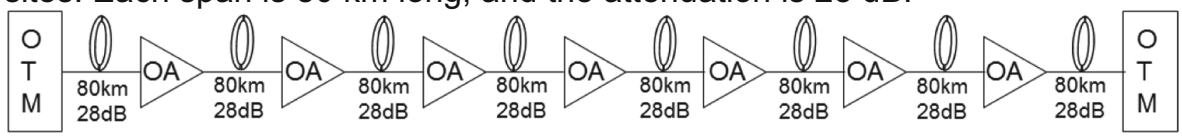


Other simulation conditions:

- The equipment rooms housing optical and electrical racks are over $1 \mathrm{~km}$ away from each other. The equipment rooms are connected using optical cables. Multiple fiber patch cords may exist in the sites. Each site has two ODF connectors. The connector loss is $2 \mathrm{~dB}(0.5 \times 4)$, the fiber loss is $0.4 \mathrm{~dB} / \mathrm{km}$, and the extra loss of pigtails is $0.5 \mathrm{~dB}$. The total loss is $3 \mathrm{~dB}$.

- The equipment rooms housing optical and electrical racks are less than $1 \mathrm{~km}$ away from each other, and are directly connected using optical fibers. There is no connector loss, fiber attenuation, or fiber layout loss. The total loss is $1 \mathrm{~dB}$. If an ODF is used to connect the equipment rooms, the connector loss is $1 \mathrm{~dB}(0.5 \times 2)$, and the fiber loss is $0.5 \mathrm{~dB}$. Considering the fiber layout loss, the total loss is $2 \mathrm{~dB}$.

\subsection{Solution-Based Simulation for Different Scenarios}

OSNR simulation for non-remote electrical racks (Table 1).

Table 1. OSNR simulation for non-remote electrical racks

\begin{tabular}{l|l|l}
\hline Simulation model & Incident optical power & Receive-end OSNR \\
\hline $8 \times 24 \mathrm{~dB}$ & $1 \mathrm{dBm}$ & $20.6 \mathrm{~dB}$ \\
\hline $8 \times 28 \mathrm{~dB}$ & $1 \mathrm{dBm}$ & $16.7 \mathrm{~dB}$ \\
\hline
\end{tabular}

\section{OSNR simulation for direct fiber connection of an electrical rack (Table 2).}

Table 2. OSNR simulation for direct fiber connection of remote deployment (loss: $<2.5 \mathrm{~dB})$

\begin{tabular}{l|l|l}
\hline Simulation model & Incident optical power & Receive-End OSNR \\
\hline $8 \times 24 \mathrm{~dB}$ & $1 \mathrm{dBm}$ & $20.6 \mathrm{~dB}$ \\
\hline $8 \times 28 \mathrm{~dB}$ & $1 \mathrm{dBm}$ & $16.7 \mathrm{~dB}$ \\
\hline
\end{tabular}

\section{OSNR simulation for direct fiber connection of an electrical rack (Table 3).}

Table 3. OSNR simulation for direct fiber connection of remote deployment (loss: $5 \mathrm{~dB}$ )

\begin{tabular}{l|l|l}
\hline Simulation model & Incident optical power & Receive-end OSNR \\
\hline $8 \times 24 \mathrm{~dB}$ & $1 \mathrm{dBm}$ & $20.4 \mathrm{~dB}$ \\
\hline $8 \times 28 \mathrm{~dB}$ & $1 \mathrm{dBm}$ & $16.6 \mathrm{~dB}$ \\
\hline
\end{tabular}


OSNR simulation for remote deployment through added OMSs (Table 4).

Table 4. OSNR simulation for remote deployment through added OMSs (added span: $10 \mathrm{~dB}$ )

\begin{tabular}{l|l|l}
\hline Simulation model & Incident optical power & Receive-end OSNR \\
\hline $8 \times 24 \mathrm{~dB}$ & $1 \mathrm{dBm}$ & $20.2 \mathrm{~dB}$ \\
\hline $8 \times 28 \mathrm{~dB}$ & $1 \mathrm{dBm}$ & $16.5 \mathrm{~dB}$ \\
\hline
\end{tabular}

\subsection{Conclusion}

The simulation results are summarized as follows:

- When the racks are directly connected using optical fibers, the attenuation is within $2.5 \mathrm{~dB}$, and the receive-end OSNR remains unchanged.

- When the racks are directly connected using optical fibers, the attenuation is greater than $2.5 \mathrm{~dB}$, which has slight impact on the OSNR but great impact on the receive optical power of the OTU boards. When the system optical power fluctuates, alarms are easily generated.

- Adding OMSs to connect racks affects the OSNR. When the OSNR margin is large, the OSNR is decreased by about $0.5 \mathrm{~dB}$. When the OSNR margin is small, the OSNR is decreased by $0.2 \mathrm{~dB}$, which still has great impact on the system.

- If the racks are near to each other and the attenuation is less than $2.5 \mathrm{~dB}$, the direct fiber connection mode is recommended, which has no impact on the OSNR.

- If the racks are far away from each other, the attenuation is greater than $2.5 \mathrm{~dB}$, and the OSNR margin is large, it is recommended that OMSs be added to connect the racks.

- If the racks are far away from each other, the attenuation is greater than $2.5 \mathrm{~dB}$, and the OSNR margin is small, remote deployment is not recommended. Instead, the equipment room and planning should be adjusted to house the racks together.

\section{Application on Live Networks}

The OTN electrical rack remote deployment solution described in this document has been applied in phase II of China Mobile's ITMC private network. The following uses the remote electrical rack deployment in the Xili equipment room in Shenzhen as an example.

\subsection{Live Network Description}

As shown in Fig. 5, the Xili equipment room has five directions. MS 5030 is connected to the Guanlan equipment room; MS 5031 and MS 5033 are connected to equipment rooms in Hong Kong to form a four-point WDM ring network; MS 5019 is connected to an equipment room in Qinghedong of Guangzhou; MS 5009 is connected to an equipment room in Ganzhou of Jiangxi province. 


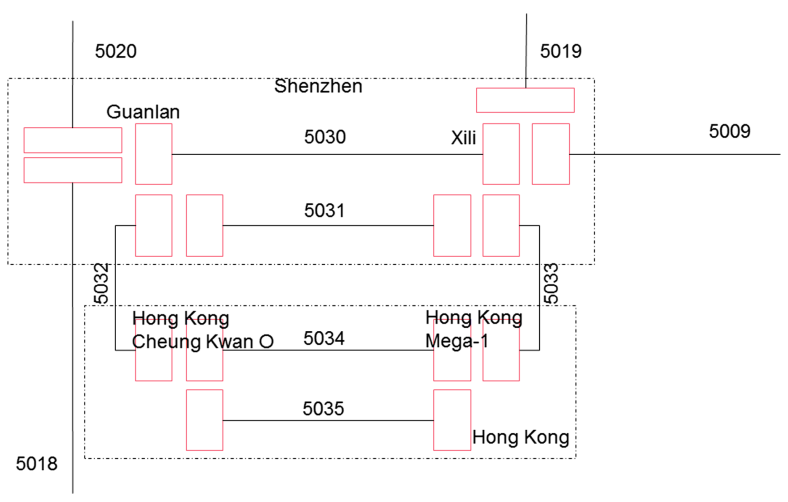

Fig. 5. Network architecture of the Xili equipment room in Shenzhen

\subsection{Solution Selection}

Table 5 lists the performance parameters, current-period performance parameters, OSNR thresholds, and OSNR margin of each MS during the network construction in phase 9.2.

Table 5. Live-network performance parameters

\begin{tabular}{l|l|l|l|l|l}
\hline MS No. & MS name & $\begin{array}{l}\text { Designed } \\
\text { OSNR }\end{array}$ & $\begin{array}{l}\text { Live-network } \\
\text { OSNR }\end{array}$ & $\begin{array}{l}\text { OSNR } \\
\text { threshold }\end{array}$ & $\begin{array}{l}\text { Current OSNR } \\
\text { margin }\end{array}$ \\
\hline 5009 & Xili - Ganzhou & 18.6 & 19 & 18.4 & 0.6 \\
\hline 5019 & $\begin{array}{l}\text { Xili } \\
- \text { Qinghedong }\end{array}$ & 21.7 & 21.2 & 18.4 & 2.8 \\
\hline 5030 & Xili - Guanlan & 31.5 & 30.3 & 18.4 & 11.9 \\
\hline 5031 & Xili - Guanlan & 26.1 & 23.3 & 18.4 & 4.9 \\
\hline 5033 & $\begin{array}{l}\text { Xili - Hong } \\
\text { Kong Mega I }\end{array}$ & 23.1 & 26 & 18.4 & 7.6 \\
\hline
\end{tabular}

According to the survey on the live network, if the direct fiber connection mode is used, the attenuation of the connection between the new electrical rack (OTU board) and the old optical rack (M40V/D40) at the local site is less than or equal to $1.5 \mathrm{~dB}$. Based on the specifications of second-generation 100G, Table 6 lists the performance changes of each MS.

MS 5009 determines the distance between the new electrical rack and the old optical rack in Xili, because among the five MSs, the OSNR margin of MS 5009 is the lowest.

Solution 1: Use optical fibers/cables for direct connection. When the secondgeneration 100G boards are used, the OSNR margin of MS 5009 is $3.4 \mathrm{~dB}$. If this solution is used, the incident optical power of the OTU boards in MS 5009 can remain unchanged, that is, the OSNR on the live network should be $19 \mathrm{~dB}$. The OSNR margin of $100 \mathrm{G}$ channels on the live network is $0.6 \mathrm{~dB}$. If the second-generation $100 \mathrm{G}$ boards 
Table 6. Performance parameters after direct fiber connection

\begin{tabular}{l|l|l|l|l|l|l}
\hline MS No. & MS name & $\begin{array}{l}\text { OSNR } \\
\text { threshold }\end{array}$ & $\begin{array}{l}\text { OSNR at } \\
1.5 \mathrm{~dB} \\
\text { connection } \\
\text { loss }\end{array}$ & $\begin{array}{l}\text { OSNR } \\
\text { margin at } \\
1.5 \mathrm{~dB} \\
\text { connection } \\
\text { loss }\end{array}$ & $\begin{array}{l}\text { Connection } \\
\text { insertion } \\
\text { loss at } \\
\text { OSNR } \\
\text { margin } \\
\text { greater than } \\
1 \mathrm{~dB}\end{array}$ & $\begin{array}{l}\text { Max. } \\
\text { insertion loss } \\
\text { supporting } \\
\text { service } \\
\text { provisioning }\end{array}$ \\
\hline 5009 & $\begin{array}{l}\text { Xili } \\
- \text { Ganzhou }\end{array}$ & 15.6 & 19 & 3.4 & 2.5 & 2.5 \\
\hline 5019 & $\begin{array}{l}\text { Xili- } \\
\text { Qinghedong }\end{array}$ & 15.6 & 21 & 5.4 & 2.5 & 2.5 \\
\hline 5030 & $\begin{array}{l}\text { Xili } \\
- \text { Guanlan }\end{array}$ & 15.6 & 30.3 & 14.7 & 2.5 & 2.5 \\
\hline 5031 & $\begin{array}{l}\text { Xili } \\
- \text { Guanlan }\end{array}$ & 15.6 & 22.1 & 6.5 & 2.5 & 2.5 \\
\hline 5033 & $\begin{array}{l}\text { Xili - Hong } \\
\text { Kong Mega I }\end{array}$ & 15.6 & 24.5 & 8.9 & 2.5 & 2.5 \\
\hline
\end{tabular}

are used for new services, the OSNR threshold of the second-generation 100G HDFEC boards is expected to be $15.6 \mathrm{~dB}$, and the OSNR margin will be $3.4 \mathrm{~dB}$.

Solution 2: Add OMSs. The new electrical rack and old electrical rack are connected through an optical rack, which is similar to an inter-office transfer ring. Simulate the scenario where the line attenuation between the new electrical rack and the old electrical rack is $10 \mathrm{~dB}$, the end-to-end OSNR from the new electrical rack in Xili to Ganzhou is $17.3 \mathrm{~dB}$, the OSNR threshold is $15.6 \mathrm{~dB}$, and the OSNR margin is $1.7 \mathrm{~dB}$.

Solution 3: Adjust the position of equipment rooms. If the new equipment room is located together with the old equipment room and the fiber distance and attenuation remain unchanged, the OSNR of each MS remains unchanged.

Based on the preceding analysis, when the equipment rooms cannot be adjusted, the actual indicators are consistent with the analysis results if solution 1 is used.

\section{Conclusions and Suggestions}

When the equipment room space and power consumption on the live network are increasingly limited, separate deployment of optical and electrical racks needs to be considered during live network planning and design, and relevant rules need to be specified in advance. This prevents implementation failures and O\&M difficulties in the future and prevents service performance from being affected by remote deployment.

Based on the comparison and analysis of remote deployment modes and scenarios, performance simulation, and live network implementation solutions, it is recommended that the remote deployment modes be selected as follows: 
- If optical and electrical racks are close to each other and the OSNR margin is large, use optical fibers to directly connect the racks or add OMSs to connect the racks.

- If optical and electrical racks are close to each other and the OSNR margin is small, use optical fibers to directly connect the racks.

- If optical and electrical racks are far away from each other and the OSNR margin is large, add OMSs to connect the racks.

- If optical and electrical racks are far away from each other and the OSNR margin is small, adjust the equipment room plan or service plan, because remote deployment is not applicable.

Open Access This chapter is licensed under the terms of the Creative Commons Attribution 4.0 International License (http://creativecommons.org/licenses/by/4.0/), which permits use, sharing, adaptation, distribution and reproduction in any medium or format, as long as you give appropriate credit to the original author(s) and the source, provide a link to the Creative Commons license and indicate if changes were made.

The images or other third party material in this chapter are included in the chapter's Creative Commons license, unless indicated otherwise in a credit line to the material. If material is not included in the chapter's Creative Commons license and your intended use is not permitted by statutory regulation or exceeds the permitted use, you will need to obtain permission directly from the copyright holder.

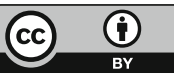

